\title{
Secretion and reversible assembly of extracellular-like matrix by enzyme-active colloidosome-based protocells
}

Khrongkhwan Akkarachaneeyakorn, Mei Li, Sean A. Davis, Stephen Mann*

Centre for Protolife Research and Centre for Organized Matter Chemistry, School of Chemistry, University of Bristol, Bristol BS8 1TS, United Kingdom

\section{Supporting Information: Figures}

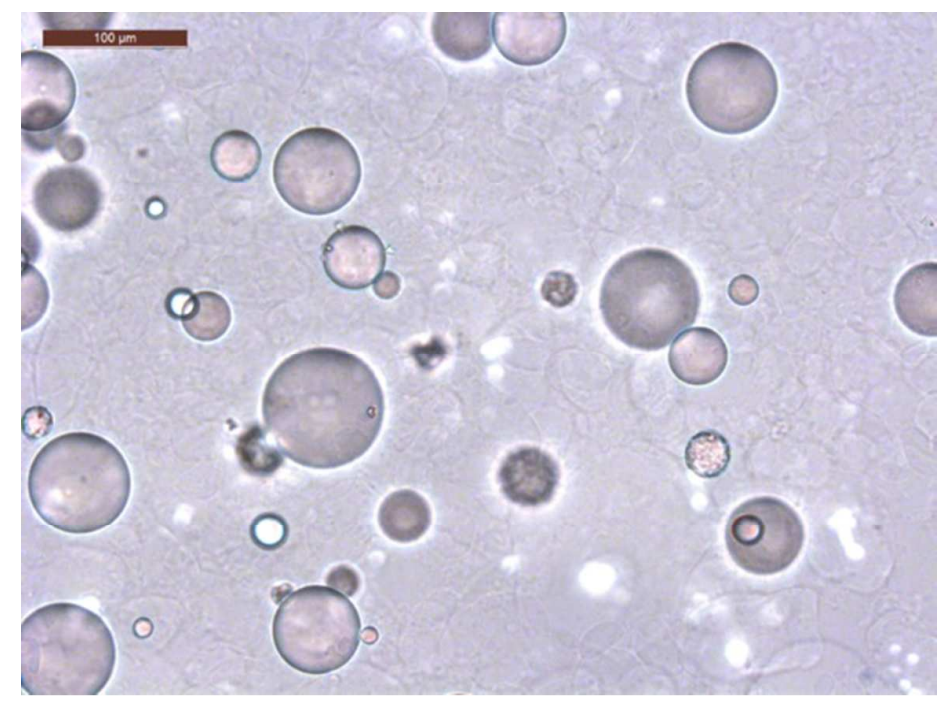

Figure S1. Optical microscopy image showing intact ALP-containing silica nanoparticle-based colloidosomes after transfer from oil into water. Scale bar $=100 \mu \mathrm{m}$
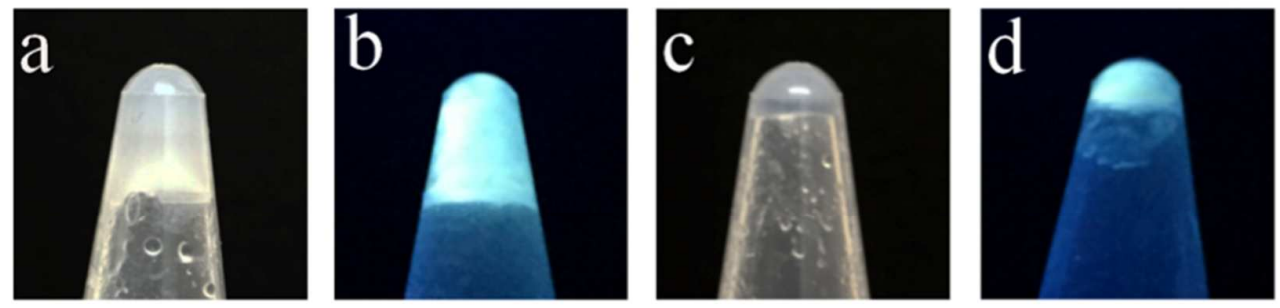

Figure S2. Photographs of Hoechst 33258-stained Fmoc-TyrOH hydrogel/colloidosome composites observed under normal light $(\mathbf{a}, \mathbf{c})$ or UV irradiation $(\mathbf{b}, \mathbf{d})$. Samples were prepared by mixing $50 \mu \mathrm{L}(\mathbf{a}, \mathbf{b})$ or $200 \mu \mathrm{L}(\mathbf{c}, \mathbf{d})$ of an aqueous dispersion of ALP-containing colloidosomes with $25 \mu \mathrm{mol}$ of Fmoc-TyrP in alkaline buffer solution and aging for 2 days. Samples are shown inverted in a plastic tube. 


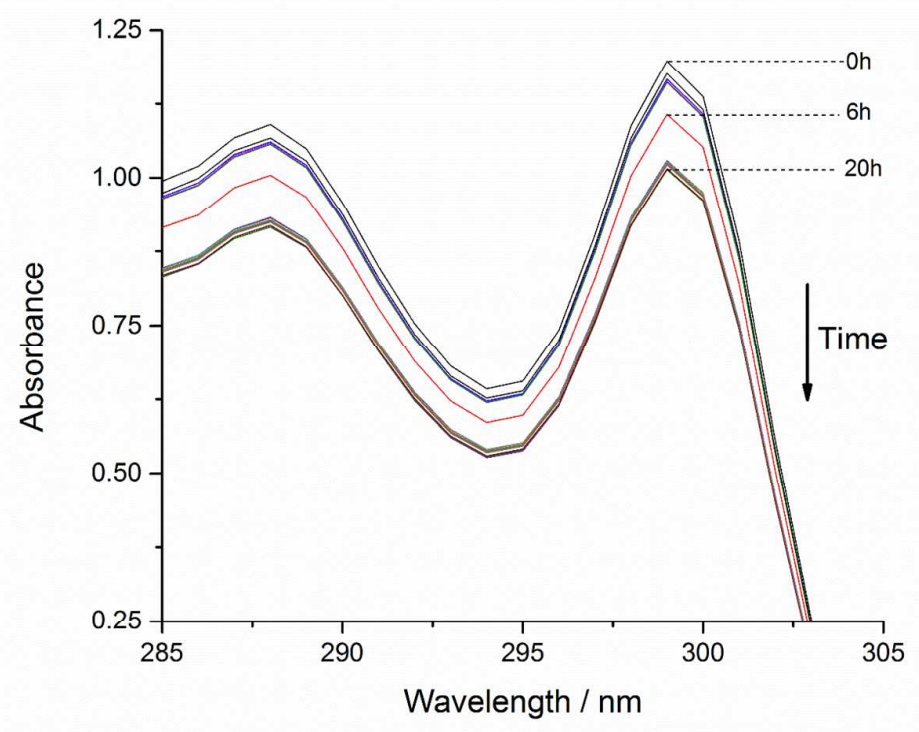

Figure S3. Time-dependent UV-vis spectra of the supernatant associated with the formation of a hydrogel/colloidosome composite. Spectra were recorded hourly for a period of $20 \mathrm{~h}$. The decrease in absorbance at 288 and $299 \mathrm{~nm}$ with time corresponds to a reduction in the concentration of Fmoc-TyrP as the dephosphorylation reaction proceeds.

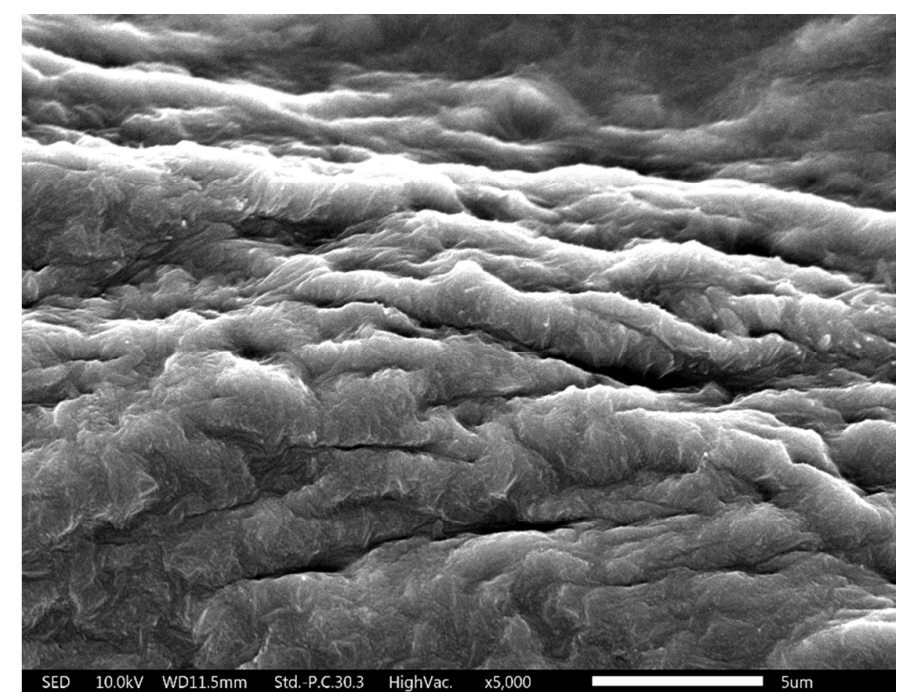

Figure S4. SEM image of Fmoc-TyrOH hydrogel prepared in bulk solution by ALP-mediated dephosphorylation of Fmoc-TyrP. Note the dense superstructure of bundled fibres. Scale bar $=5 \mu \mathrm{m}$. 

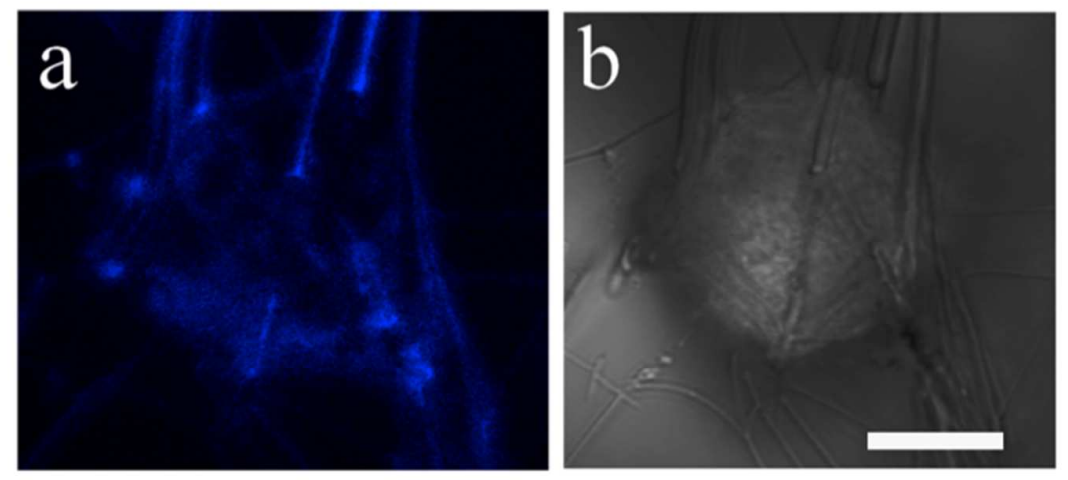

Figure S5. Confocal fluorescence (a) and corresponding optical (b) microscopy images showing single colloidosome and associated Fmoc-TyrOH nanofilaments stained with the blue fluorescent dye, Hoechst 33258. Sample was aged for $20 \mathrm{~h}$. Note the virtual absence of staining within the protocell after this time period. Scale bar $=30 \mu \mathrm{m}$.
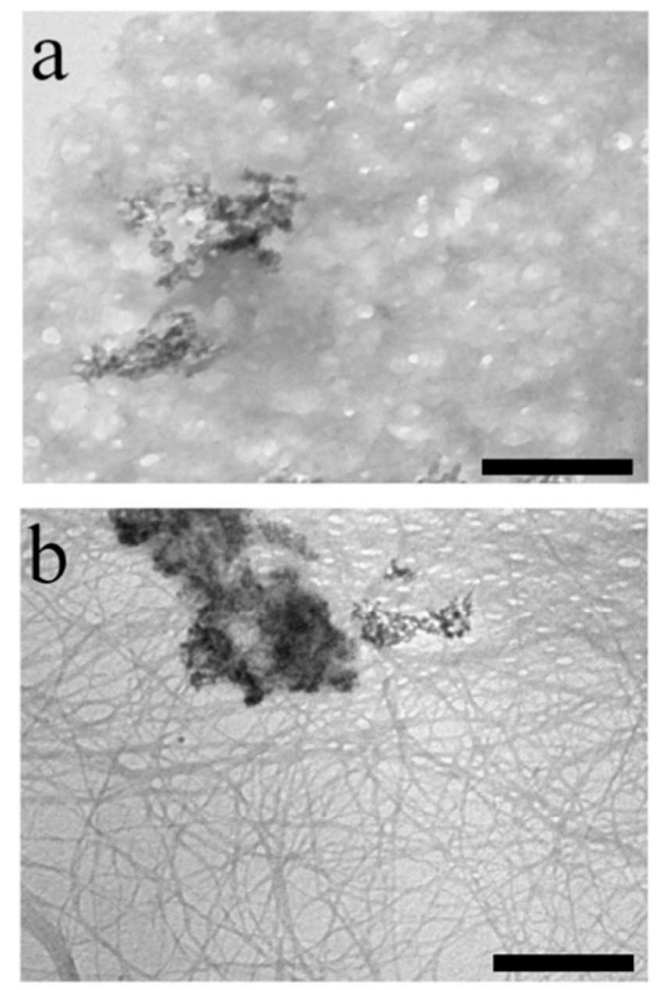

Figure S6. TEM images of fractured unstained sample of a Fmoc-TyrOH hydrogel/colloidosome composite isolated after (a) 5 and (b) 20 h showing transformation from internal unstructured hydrogel to dense network of externally located nanofilaments. Scale bars $=0.25$ and (b) $1 \mu \mathrm{m}$ in (a) and (b), respectively. 


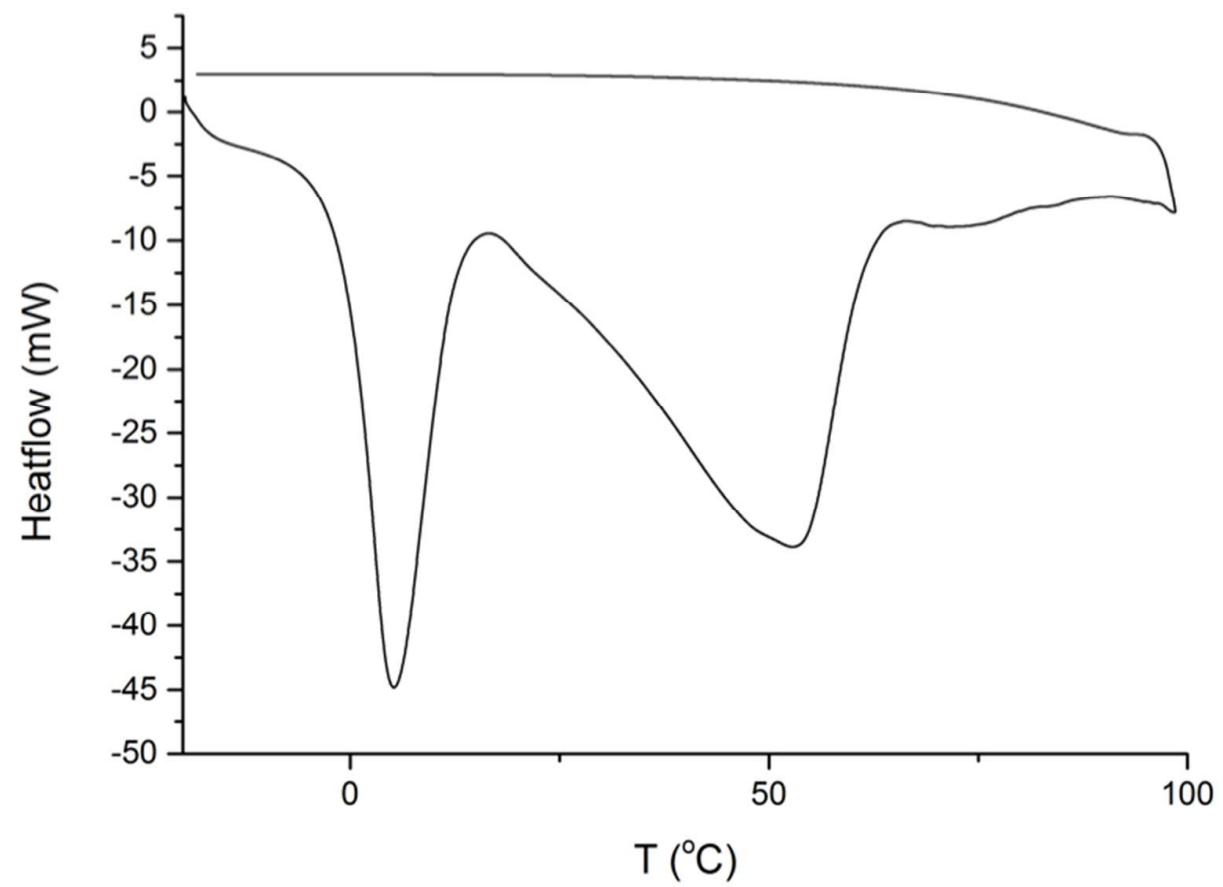

Figure S7. DSC thermogram of Fmoc-TyrOH hydrogel prepared from ALP-mediated dephosphorylation of Fmoc-TyrP showing broad gel to sol transition centred at $53^{\circ} \mathrm{C}$. 

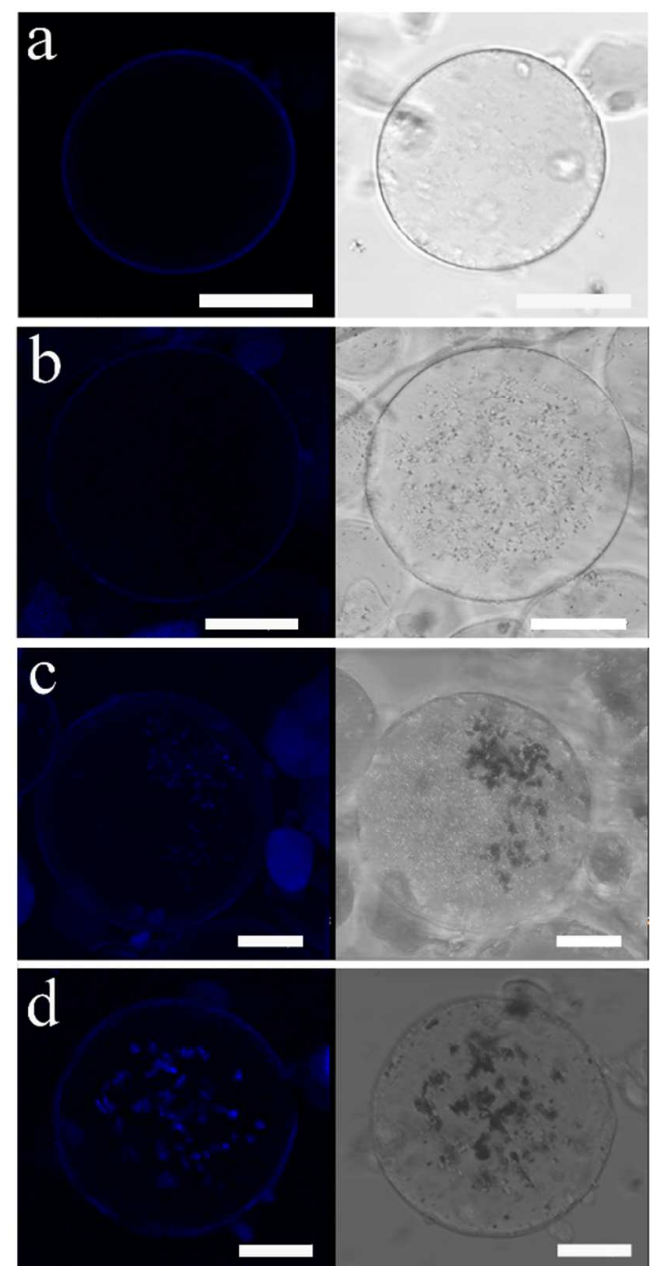

Figure S8. Fluorescence and corresponding bright field confocal microscopy images of single ALP-containing colloidosomes after addition of Fmoc-TyrP and aging at room temperature for $20 \mathrm{~h}$, heating to $60^{\circ} \mathrm{C}$ for $2 \mathrm{~h}$, and cooling to room temperature and left for (a) 0 (b) 3 , (c) 4 , and (d) $5 \mathrm{~h}$; scale $=30 \mu \mathrm{m}$. 


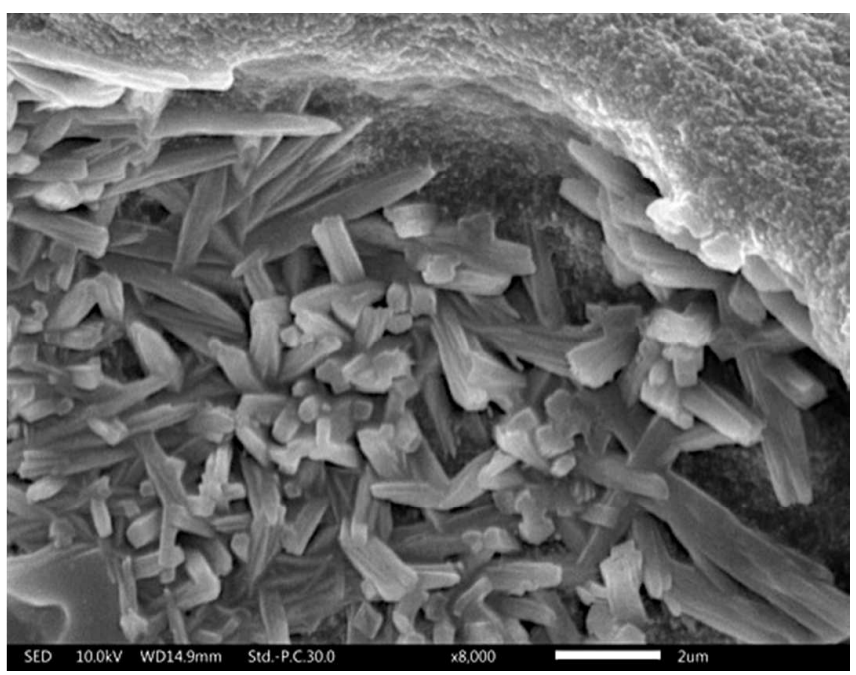

Figure S9. SEM image of fractured colloidosome showing densely packed reassembled bundles of Fmoc-TyrOH filaments produced by cooling a sample of the hydrogel/protocell composite from $60^{\circ} \mathrm{C}$ to room temperature and aging the mixture for $20 \mathrm{~h}$. Scale bar $=2 \mu \mathrm{m}$.
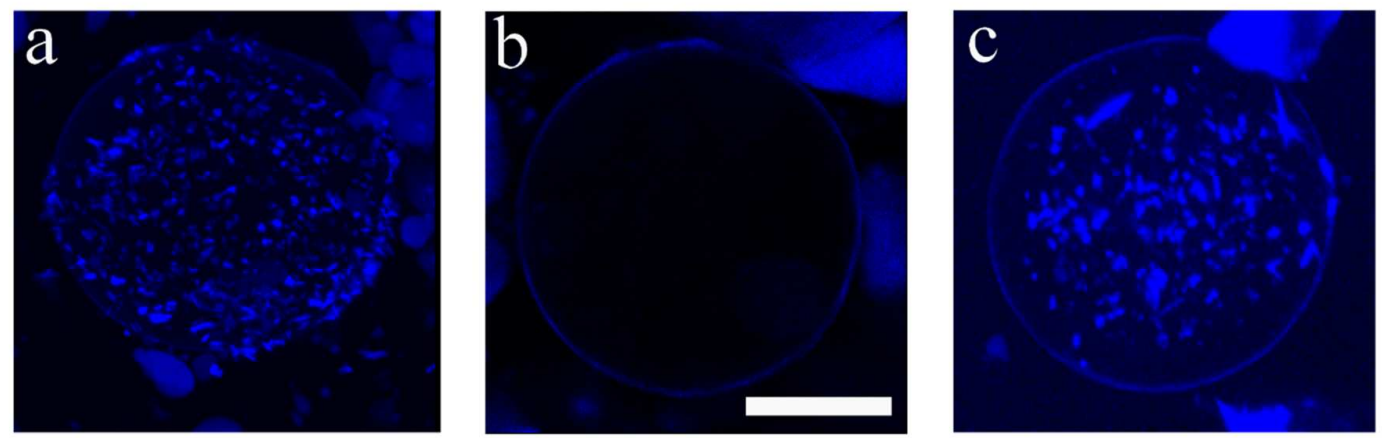

Figure S10. Fluorescence images of individual protocells showing (a) internalized reassembly of hydrogel matrix after disassembly at $60^{\circ} \mathrm{C}$ followed by cooling to room temperature, (b) same sample after reheating to $60^{\circ} \mathrm{C}$ showing disassembly of reassembled hydrogel, and (c) cooling to room temperature displaying next cycle of reassembly specifically in the colloidosome interior. Scale bar for all images, $40 \mu \mathrm{m}$. 

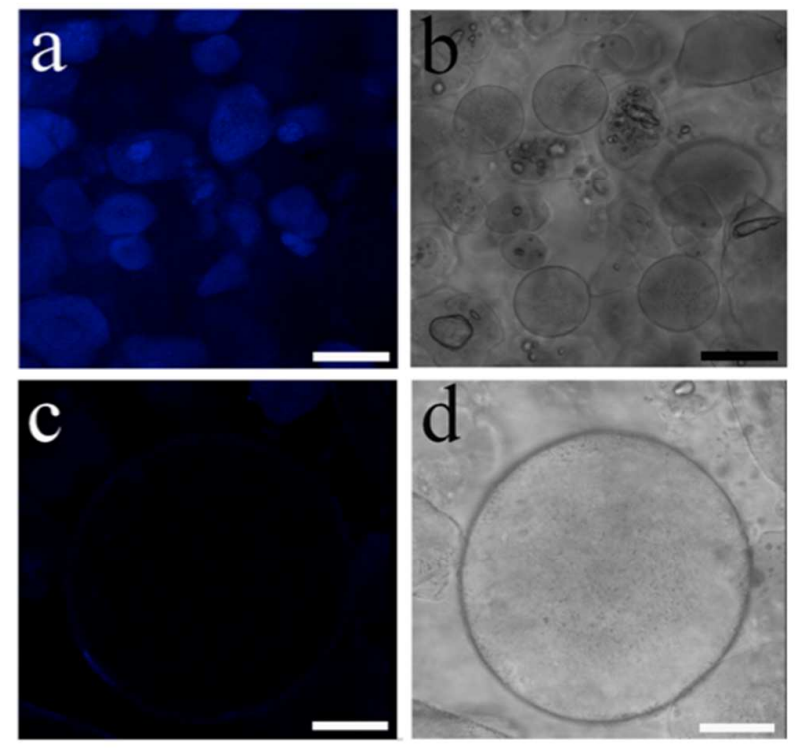

Figure S11. $(\mathbf{a}, \mathbf{b})$ Fluorescence and corresponding bright field images of a population of colloidosomes containing EDTA-deactivated ALP after addition of the protocell dispersion to a preformed Fmoc-TyrOH hydrogel produced in bulk solution, and the mixture heated above the gel-sol transition temperature and then cooled to room temperature overnight. No blue fluorescence corresponding to the reassembled hydrogel is observed within the colloidosome micro-compartments. Scale bar $=50 \mu \mathrm{m}$ (c,d) Images from samples prepared as above but by addition of water-filled (no ALP) colloidosomes to the preformed hydrogel showing absence of Fmoc-TyrOH nanofilaments in the protocell interior. Scale bar $=20 \mu \mathrm{m}$.
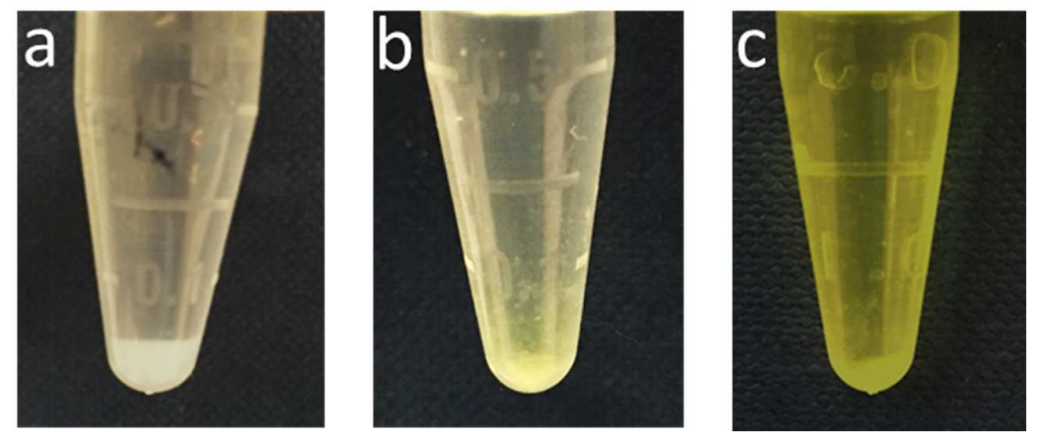

Figure S12. (a) Photograph of sample tube containing hydrogel-filled ALP-containing colloidosomes after disassembly at $60^{\circ} \mathrm{C}$ followed by cooling to room temperature for $20 \mathrm{~h}$. $(b, c)$ Same sample after addition of $p$-nitrophenylphosphate (pNPP) and after $1 \mathrm{~h}$ (b) and $8 \mathrm{~h}$ (c) showing development of yellow colouration due to formation of the dephosphorylated product, $p$-nitrophenyl (pNP) within the colloidosomes and diffusion into the supernatant. 


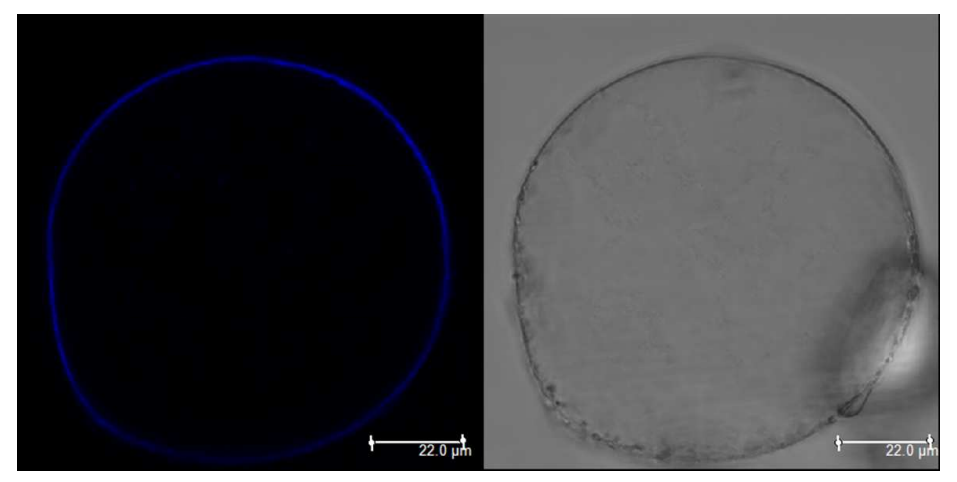

Figure S13. Fluorescence (left) and optical (right) microscopy images of a single ALP-free colloidosome incubated in an aqueous solution of Fmoc-TyrP and showing the absence of hydrogel formation. The background blue fluorescence originates from Hoechst 33258 molecules adventiously absorbed onto the silica nanoparticles of the colloidosome membrane. Scale bar $=22 \mu \mathrm{m}$. 